\title{
On the suitability of direct application of acoustic theory to time-resolved tomographic PIV tested by DNS for low Mach number jet flows
}

\author{
Peter Moore*, Daniele Violato ${ }^{\dagger}$, Kristof Bryon $^{\ddagger}$ and Fulvio Scarano ${ }^{\S}$
}

In this paper, we investigate the minimum temporal requirements, spatial domain and spatial resolution requirements in order to make acoustic predictions by means of acoustic analogies, for low speed jet flows, by means of numerical simulations. This work is done to demonstrate the feasibility of indirect acoustic extrapolation from recent experiments undertaken by us and to develop robust techniques. For this paper we consider Direct Numerical Simulation of incompressible jets flows of Reynolds number 2,500 and Reynolds number 5,000 jets. Comparison of flow statistics and visualizations confirms that the simulations are representative of the jets obtained in our experiments. Lighthill's and Powell's aerocaoustic analogies are applied and demonstrated to yield indirect acoustic predictions of these jets, especially at $90^{\circ}$ to the flow axis, where the coordinate origin dependency of Powell's acoustic analogy is minimal. Finally we investigate the robustness of these indirect acoustic predictions when the DNS simulation data is reduced in temporal and spatial extent and resolution to those currently achievable by our Tomographic PIV setup. The results indicate that our current measurement capabilities are suitable for obtaining acoustic spectra up to a Strouhal number of 1.0. Moreover, in order to improve the spectral cut-off, it is preferable to increase the spatial resolution of the measurements at the expense of domain size and temporal resolution.

\section{Introduction}

The possibility of experimentally obtaining the full aeroacoustic source by means of PIV is relevant to aerocaoustic testing technologies in that it would allow the study of aeroacoustic emissions in wind tunnels other than anechoic, or even in water. Moreover, it allows for academic investigations of noise mechanisms at conditions much closer to real-world configurations (especially Reynolds number range, accurate boundary conditions and the inclusion of complex geometries like Chevron nozzles).

Time-resolved tomographic PIV appears at the outset to meet the necessary criteria for an aeroacoustic study: providing a complete time-resolved 3-D flow field. However, the achievable spatial resolution is restrictive, and the effects of measurement uncertainty ${ }^{1}$ need to be quantified for aeroacoustic purposes. Still, significant progress in the use of time resolved PIV as an acoustic source has been made in recent years.

For flows involving the interaction of large vortices with solid boundaries, the use of acoustic analogies with time-resolved PIV data appears to be quite robust. For example, in the case of self-sustained cavity oscillations, both Koschatzky et al. (2009) ${ }^{2}$ and Haigermoser $(2009)^{3}$ apply Curle's acoustic analogy ${ }^{4}$ to time-resolved PIV data and respectively find good agreement with microphone measurements and theory. Similarly, the case of a an airfoil in a fluctuating flow (such as a Kármán vortex street) is highly efficient. In this case, Lorenzoni et al. (2009) ${ }^{5}$ and Moore et al. (2009) ${ }^{6}$ find good agreement between experimental data and predictions of various methods based on acoustic analogies when applied to time resolved PIV data.

The case of jet flow is certainly more challenging, particularly as the acoustic source in this case is far less efficient, being of quadrupole nature. Nevertheless, progress has also been made in this direction. Wernet (2007) ${ }^{7}$ investigated high speed jet flows using planar time-resolved PIV. He was able to measure a significant portion of the two-point space time velocity correlation tensor that is important to aeroacoustic theory.

\footnotetext{
*Postdoc, Delft University of Technology, Aerodynamics department. Kluyverweg 2, 2629 HT, Delft, the Netherlands.

${ }^{\dagger} \mathrm{PhD}$ student, Delft University of Technology, Aerodynamics department. Kluyverweg 2, 2629 HT, Delft, the Netherlands.

$\ddagger$ Master student, Delft University of Technology, Aerodynamics department. Kluyverweg 2, 2629 HT, Delft, the Netherlands.

$\S$ Professor, Delft University of Technology, Aerodynamics department. Kluyverweg 2, 2629 HT, Delft, the Netherlands.
} 
Schram and Hirshberg (2003) ${ }^{8}$ and Schram et al. (2005) ${ }^{9}$ used phase-locked PIV to evaluate the acoustic emissions associated with leap-frogging vortices in the roll-up of the shear layer in a circular round jet. In particular, they noted that formulations enforcing conservative properties were considerable more robust in providing estimates of the acoustic emissions.

The time-resolved 3-D tomographic PIV approach in our project can be considered a powerful generalization of the approach of Schram and Hirshberg (2003) ${ }^{8}$ and Schram et al. (2005) ${ }^{9}$. However, as our initial experiments were performed in water, direct experimental validation of this approach (which might normally be achieved by comparison with microphones) is difficult. Here we develop and test through Direct Numerical Simulation the techniques used to obtain acoustic information about these flows, as they might be obtained from experimental input data.

There are several purposes to using an experimental approach. The direct application of acoustic theory to experimental PIV data, which enable the ability to quickly and accurately investigate flows at a range of Reynolds numbers for real configurations and obtain fairly complete flow data. It is specially gratifying that PIV experiments may be able to reliably obtain information about the acoustic source and flow from complex nozzle types, such as Chevrons, which is difficult using numerical simulation without a large degree of turbulence modeling.

Moreover, the investigation of fundamental source issues may be suitable to PIV approaches. For example, Stromberg et al. (1980) ${ }^{10}$ identified vortex pairing as a dominant acoustic source in low Reynolds number high speed jet flows. However, Jordan and Gervais (2008) ${ }^{11}$ suggest that at higher Reynolds numbers, vortex pairing does not constitute the dominant mechanism in unexcited, high Reynolds number flows. Ultimately, it is envisaged that the development of these experimental techniques will provide a valuable tool to investigate issues such as that raised by Jordan and Gervais $(2008)^{11}$.

This paper attempts to answer the following questions: "Can experimental 3-D time-resolved Tomographic PIV data in principle be used to make accurate acoustic predictions for low speed jet flows?". The smaller questions that comprise this are "How badly are acoustic predictions affected by experimental uncertainty and domain truncation?", and "Are the spatial resolutions good enough to capture at least the low frequency content, for a range of Reynolds numbers?".

\section{Description of the experiment}

The experiment consists of a hydrostatically driven vertical water jet for time-resolved tomographic PIV. Experiments are performed in an octagonal Plexiglass TM tank, that allows extensive optical access. The jet diameter is $10 \mathrm{~mm}$ and the flow speed ranges from $0.1 \mathrm{~m} / \mathrm{s}$ to $2 \mathrm{~m} / \mathrm{s}$ with corresponding Reynolds numbers from 1,000 to 20,000 in the current configuration. For the purposes of jet characterization, planar PIV measurements of the center-plane jet cross-section are taken, while for aeroacoustics purposes, a tomographic volume of the jet is reconstructed.

In the visualizations in this abstract, the experimental data corresponds to a Reynolds number of 5,000. The field sections individually visualized were $32 \times 32 \times 130 \mathrm{~mm}^{3}$, and, depending on the parameters of the tomographic reconstruction, a vector resolution of 20,-25 vectors per $D_{j}$ is achieved. More details of the experiments are given by

Table 1. Details of the experiments

\begin{tabular}{cccccc}
\hline \hline Experiment name & $\operatorname{Re} \#$ & $B=\frac{R_{j}}{\theta}$ & $D$ & $U_{j}$ & $\Delta t \frac{U}{D_{j}}$ \\
\hline JExp2500 & 2,500 & 8.0 & $0.010 \mathrm{~m}$ & $0.20 \mathrm{~m} / \mathrm{s}$ & 0.040 \\
JExp5000 & 5,000 & 8.0 & $0.010 \mathrm{~m}$ & $0.41 \mathrm{~m} / \mathrm{s}$ & 0.041 \\
\hline
\end{tabular}

Violato et al. ${ }^{12}$. Note that these spatial resolutions are quite similar to what are typically achieve by LES calculations such as that of Gröschel et al.(2005) ${ }^{13}$. The fundamental differences are that the experiments can resolve any desired portion of the flow field and disregard the rest, while the experiments have measurement uncertainty and LES techniques have model and numerical uncertainty. 


\section{Description of the DNS}

The incompressible Navier-Stokes equations with uniform density are solved through out the flow domain. The flow is solved in a spherical coordinate system using a standard staggered arrangement of the velocity vectors. The equations are solved in space with a second-order finite difference scheme (and spectrally in the axisymmetric dimension) and integrated in time with a second-order Adams-Bashforth scheme. Details of the numerical scheme and boundary condition implementation is given by Boersma et al. (1998) ${ }^{14}$.

Two numerical simulations were completed. One with Reynolds number 2,500 and one with Reynolds number 5,000. The inlet nozzle is not explicitly included, rather, the following analytic inflow profile corresponding to the measured downstream mean velocity profile is used

$$
U_{\text {in }}=0.5\left(1+\tanh \left[0.25 \frac{R_{j}}{\theta}\left(\frac{R_{j}}{r}-\frac{r}{R_{j}}\right)\right]\right) .
$$

This profile corresponds to the type II inflow profile identified by Michalke (1984) ${ }^{15}$. In order to induce instabilities in the jet, turbulence is added by means of random modifications of the jet shear layer thickness $\theta$. The method used is essentially that used by Freund $(2001)^{16}$ for a compressible subsonic jet, but was found to be highly effective for the case here of the incompressible jet flow. The value for $B=\frac{R_{j}}{\theta}$ was found by matching with the radial velocity profile of the completed water jet simulations just downstream of the nozzle location where PIV data was available. The values for $B$ and the grid resolutions for the two simulations are given in the table 2 .

Table 2. Details of the simulations

\begin{tabular}{cccccc}
\hline \hline Simulation name & $\operatorname{Re} \#$ & $B=\frac{R_{j}}{\theta}$ & $N_{r}$ & $N_{\phi}$ & $N_{\theta}$ \\
\hline JNS2500 & 2,500 & 8.0 & 384 & 120 & 64 \\
JNS5000 & 5,000 & 8.0 & 512 & 200 & 96 \\
\hline
\end{tabular}

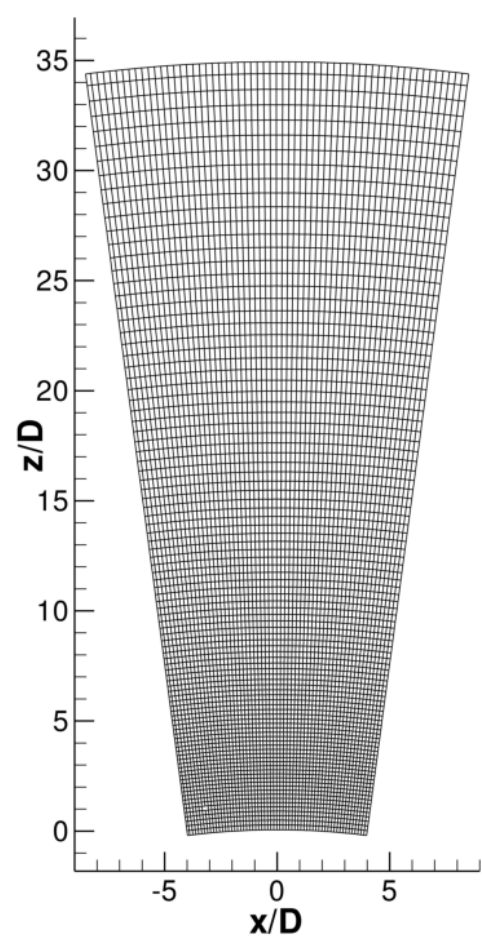

(a) JNS2500

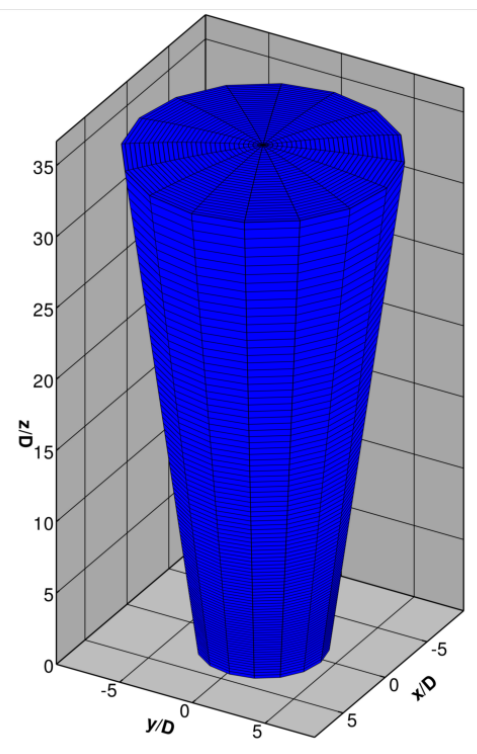

(b) JNS2500

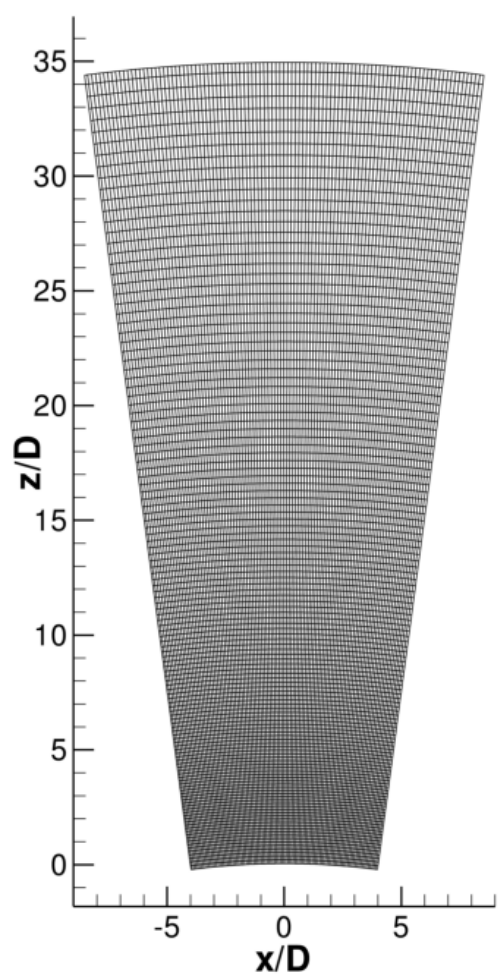

(b) JNS5000

Figure 1. Computational grids with every 4-th grid point shown with (a) 2-D slice (b) 3-D view (c) 2-D slice.

The JNS2500 simulation was run for a time of $194 D_{j} / U_{j}$ to ensure the jet was no longer transient. The full velocity and vorticity flow data was then stored at time steps separated by $0.025 D_{j} / U_{j}$ for a time of $50 D_{j} / U_{j}$. Similarly, 
the JNS5000 simulation was run initially for a time of $185 D_{j} / U_{j}$, with flow data stored with the same temporal separation as for JNS2500, for a time of $42 D_{j} / U_{j}$. The data was stored with single order precision to reduce disk usage (although all calculations are performed in double precision).

\section{Acoustic predictions}

Acoustic results are obtained by the application of Lighthill's ${ }^{17}$ and Powell's ${ }^{18}$ acoustic analogies. The form of Lighthill's acoustic analogy used is

$$
p(\boldsymbol{x}, t)=\left.\frac{\rho c_{0}^{2} M^{4}}{4 \pi R / D_{j}} \frac{\partial^{2}}{\partial t^{2}} \int_{v} \frac{(\boldsymbol{x} \cdot \boldsymbol{u})^{2}}{|\boldsymbol{X}|^{3}}\right|_{t^{*}} d^{3} \boldsymbol{y}
$$

while for Powell's analogy, the form directly used is

$$
p(\boldsymbol{x}, t)=-\left.\frac{\rho c_{0}^{2} M^{4}}{4 \pi R / D_{j}} \frac{\partial^{2}}{\partial t^{2}} \int_{v} \frac{(\boldsymbol{x} \cdot \boldsymbol{y}) \boldsymbol{x} \cdot(\boldsymbol{\omega} \times \boldsymbol{v})}{|\boldsymbol{X}|^{3}}\right|_{t^{*}} d^{3} \boldsymbol{y},
$$

where the following normalizations

$$
\begin{aligned}
& \boldsymbol{x}=\frac{\boldsymbol{x}^{o}}{R}, \\
& \boldsymbol{y}=\frac{\boldsymbol{y}^{\boldsymbol{o}}}{D_{j}}, \\
& \boldsymbol{u}=\frac{\boldsymbol{u}^{o}}{U_{j}}, \\
& \boldsymbol{\omega}=\frac{\boldsymbol{\omega}^{o} D_{j}}{U_{j}}, \\
& \begin{array}{l}
\boldsymbol{t}=\frac{t^{o} \boldsymbol{U}_{j}}{D_{j}}, \\
p=\frac{p^{o}}{\rho c_{0}^{2}}
\end{array}
\end{aligned}
$$

are used to convert from the originally quantities of physical distance, $\boldsymbol{x}^{o}$, jet scale, $\boldsymbol{y}^{o}$, time, $t^{o}$, velocity, $\boldsymbol{u}^{o}$, vorticity, $\boldsymbol{\omega}^{\boldsymbol{o}}$ and pressure $p^{o}$ to normalized quantities, where $R$ is the magnitude of the distance of the observer from the jet nozzle, $D_{j}$ is the nozzle diameter, $c_{0}$ is the speed of sound and $U_{j}$ is the jet exit velocity. Additionally,the acoustic Mach number $M=\frac{U_{j}}{c_{0}}$ and the flow density $\rho$ are introduced. These forms are the far-field compact source approximations, valid at the low Mach numbers considered here, so that $t=t^{*}+\mathrm{M} \frac{R}{D_{j}}$ gives the temporal lag between listener and emission.

The application of these analogies is not straight forward. These analogies are derived for an infinite spatial domain, where in this case, the jet flows are computed on a finite domain and exhibit a mean flow convection at both the inflow and outflow, where, particularly at the outflow, vortical structures are convected across. For Lighthill's acoustic analogy, spurious noise can result from the arbitrary truncation of convecting flow structures ${ }^{19}$ while for Powell's acoustic analogy, spurious noise is generated by a coordinate system dependence when the integration domain is finite ${ }^{20,21}$. Not that Wang et al. (1996) ${ }^{19}$ proposed a boundary correction to account for convecting sources across the integration domain for Lighthill's acoustic analogy (assuming frozen eddy convection), while Avital et al. (2008) ${ }^{21}$ proposed a similar correction for the domain dependence of Powell's acoustic analogies.

Typically if the downstream domain length is large enough, then the vortical structures are sufficiently weak at the outflow side of the integration side as to not generate acoustic noise. When the downstream domain can not be located sufficiently far downstream, then a correction such as Wang et al. (1996) ${ }^{19}$ can be used, or, more simply a windowing procedure such as that suggested by Obrist and Klesier (2007) ${ }^{22}$ (who are certainly not the first). While such a technique is simple, the technique can not be extended to Powell's acoustic analogy (for emission angles that are not perpendicular to the jet axis) because the coordinate system dependency is not resolved.

In this paper, equations $(3,2)$ are both directly solved in the stated form (rather than using a spectral version). The equations are solved using the second order rectangular method quadrature, as higher order methods can lead to spurious noise effects. For some of the calculations, when "windowing" is specified then the integrand was smoothly weighted by a sinusoidal function near the edges of the integration domain ${ }^{22}$. The window size was always $3 D_{j}$ width at the inflow and outflow sides, and $0.5 D_{j}$ on the sides that run parallel to the flow axis. The windowing procedure was used as a quick means to test if truncation was generating spurious noise. Most of the acoustic results are presented in a spectral manner. These results were obtained by a Fast Fourier Transform of $f_{w}(t)$ obtained using equation (5): which multiplies the original signal $f(t)$ by a temporal windowing function.

$$
f_{w}(t)=0.5\left(\tanh \left(5.0 \frac{t-t_{0.05}}{t_{0.05}-t_{0}}\right)-\tanh \left(5.0 \frac{t-t_{0.95}}{t_{1.0}-t_{0.95}}\right)\right) f(t),
$$

where for a signal of length $T, t_{n}=T * n$. 


\section{General results}

\section{A. Visualizations}

The simulation data and experimental data were both visualized by iso-surfaces of the vorticity magnitude for Reynolds number 2,500 in figure 2, and for Reynolds number 5,000 in figure 3. The Reynolds 2,500 jets look qualitatively

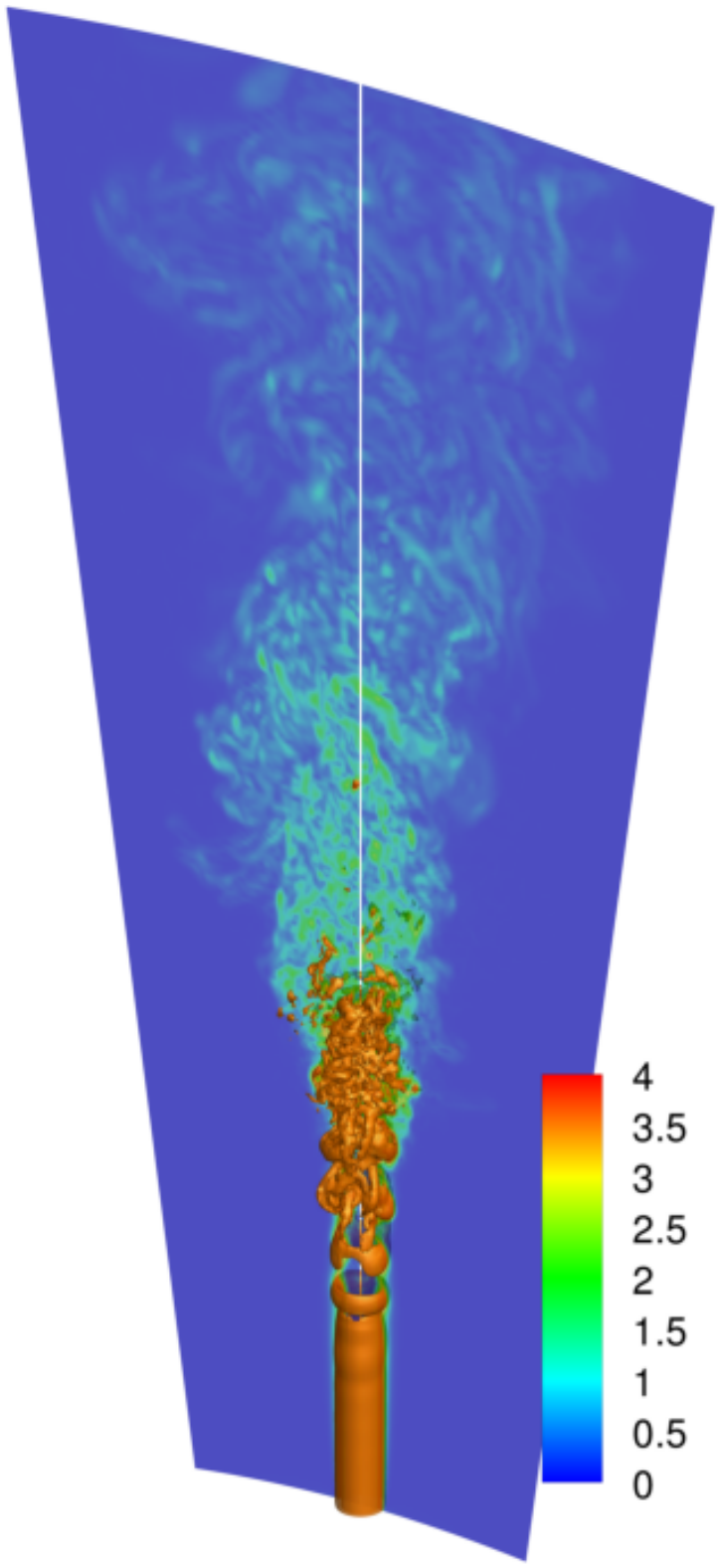

(a) JNS2500

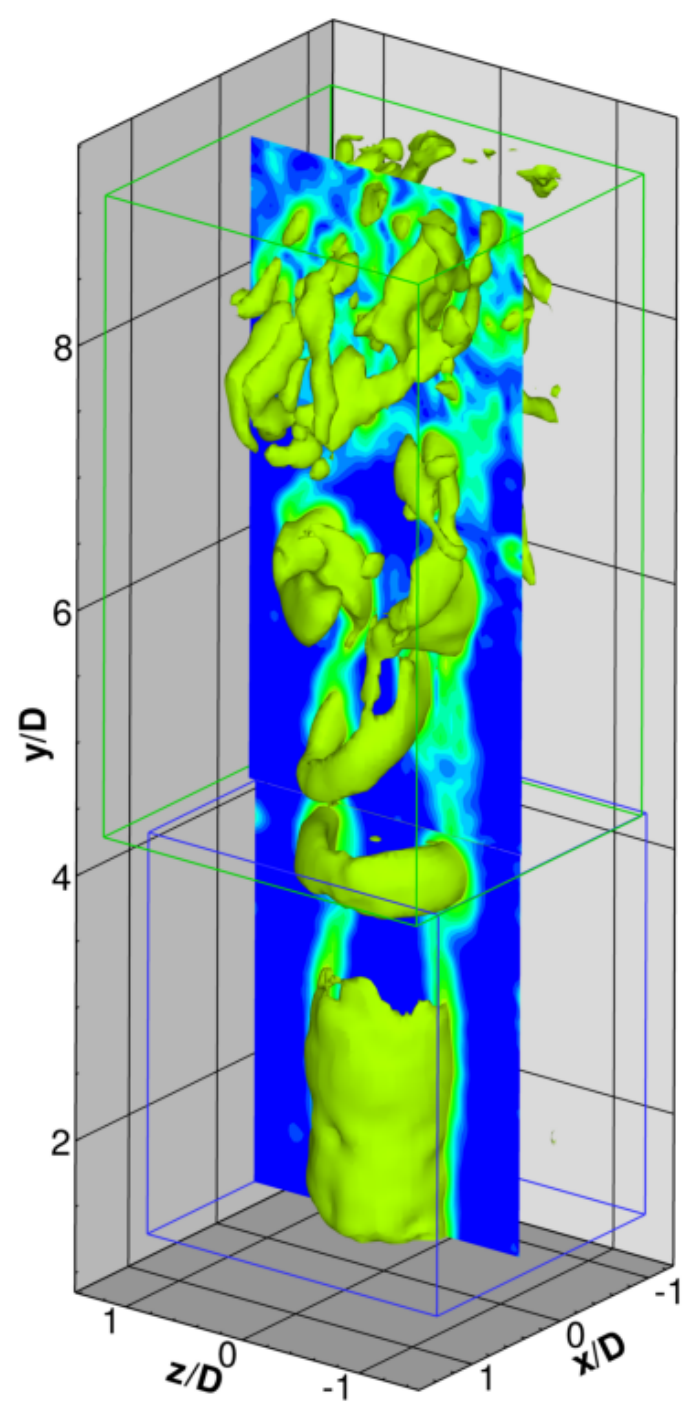

(b) JExp2500

Figure 2. Iso-surface of vorticity magnitude in three dimensions for (a) JNS2500 with $\frac{\left|\omega^{\circ}\right| D_{j}}{U_{j}}=3.5$ and (b) JExp2500 with $\frac{\left|\omega^{o}\right| D_{j}}{U_{j}}=3.5$. Not that the blue and green boxes in (b) surround data obtained at different times, and have been joined together to show a more complete 3-D jet image.

similar, with just one or two vortex rings forming towards the end of the jet potential core before their sudden collapse. Downstream, elongated structures form that mix together and convect with the flow. For the Reynolds number 5,000 jets, several distinct vortex rings are present in each case before the collapse at the end of the potential core. In the simulation, the early rings are tilted compared with experimental jet. Down stream, the structures are noticeably smaller in each each of the jets, when compared with the experimental data. While this is expected for higher Reynolds number flows, grid resolution limitations surely plays a roll at the higher Reynolds number for both the experimental and numerical results. 


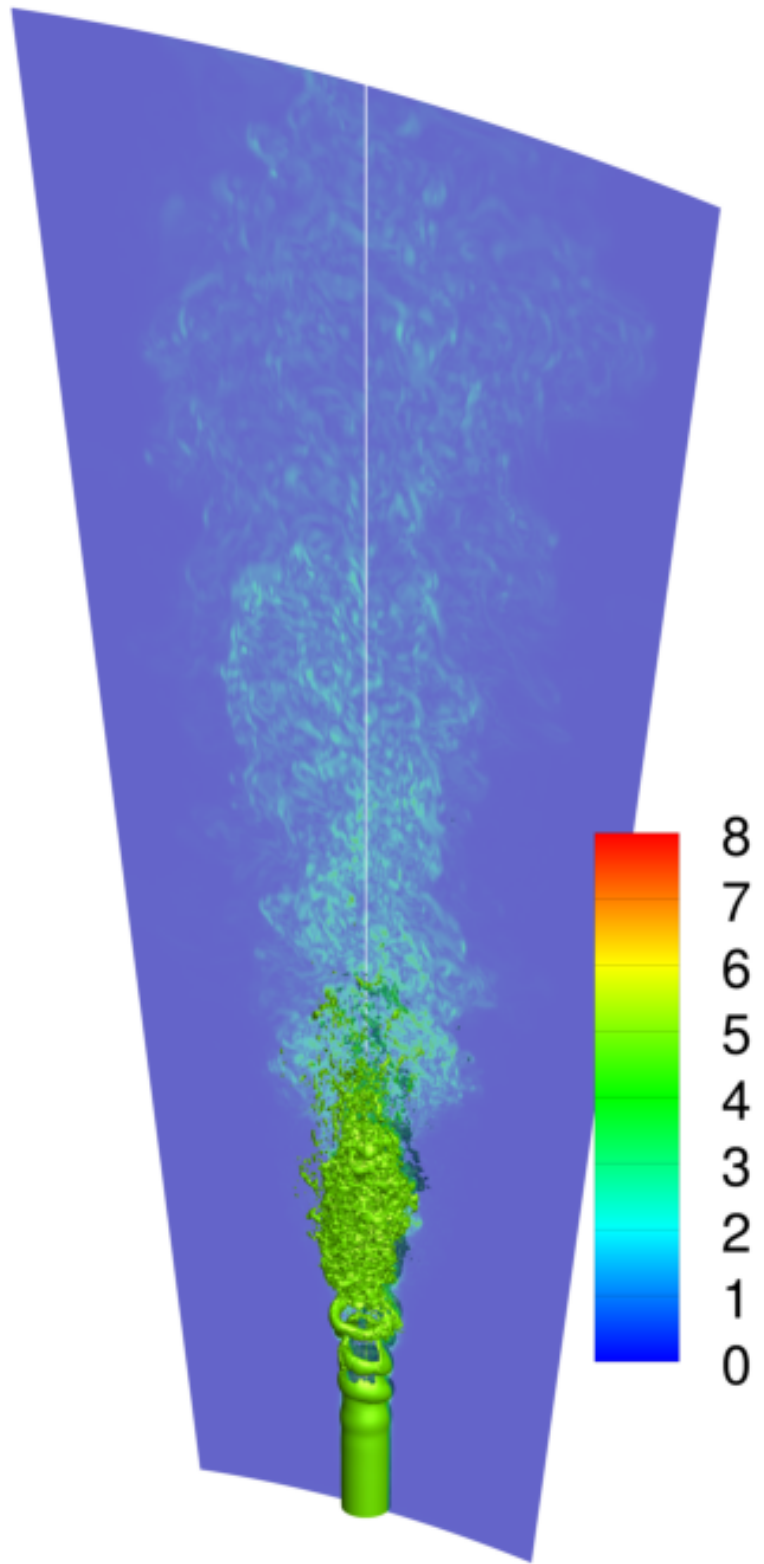

(a) JNS5000

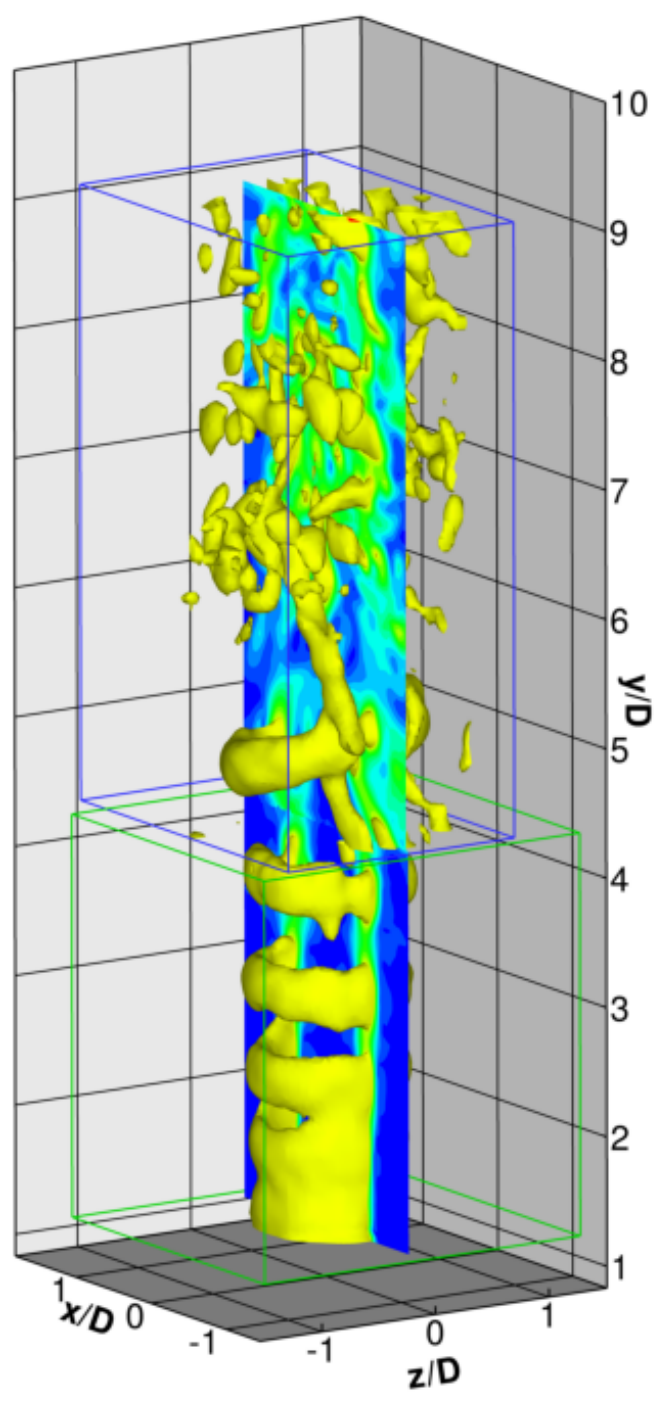

(b) JExp5000

Figure 3. Iso-surface of vorticity magnitude in three dimensions for (a) JNS5000 with $\frac{\left|\omega^{o}\right| D_{j}}{U_{j}}=6.0$ and (b) JExp5000 with $\frac{\left|\omega^{o}\right| D_{j}}{U_{j}}=3.7$. 


\section{B. Mean flow results}

In figure 4 (a), we compare the mean axial velocity and RMS of the axial velocity fluctuations for various jets The

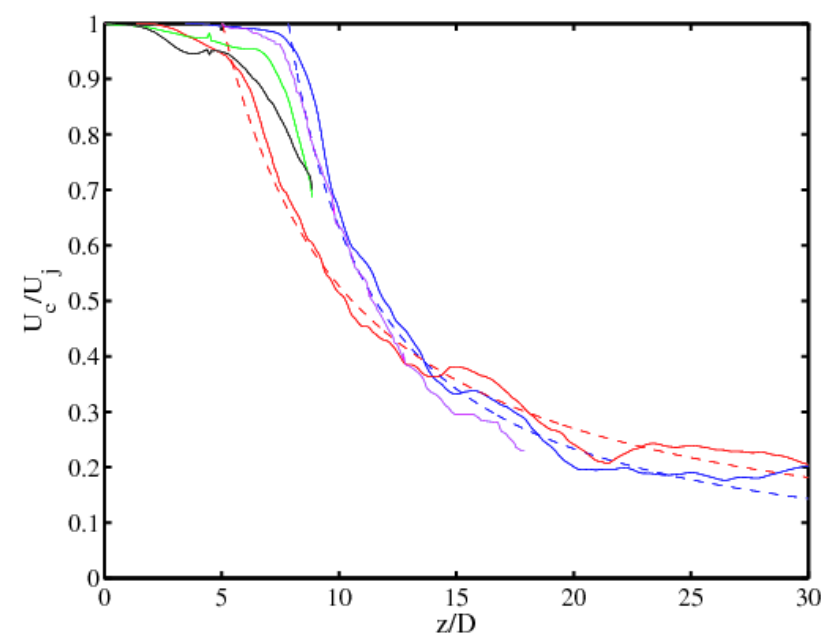

(a)

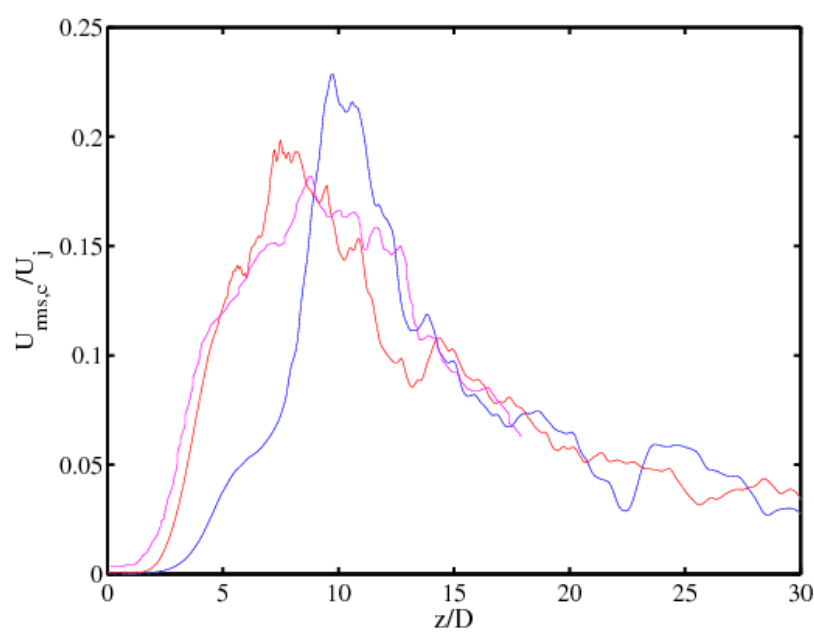

(b)

Figure 4. Centreline profile of (a) mean axial velocity and (b) axial velocity RMS. Blue line: JNS2500, red: JNS5000, green: JExp2500, black: JExp5000, magnenta: Reynolds number 6,000 incompressible simulation of Avital et al. (2008) ${ }^{21}$.

results of the simulation and experiment are consistent with faster centerline decay of the jet with decreasing Reynolds number, while for the simulation with higher peak axial velocity RMS with decreasing Reynolds number.

\section{Acoustic results}

In figure 5, we show the acoustic spectra for Lighthill's and Powell's acoustic analogies at a radiation angle of $90^{\circ}$ with respect to the flow axis, along with the domain of the acoustic integrations for JNS2500. In figure 6, we show the same, but for JNS5000. The spectra for JNS2500 have peak emission at a Strouhal number of 0.2. For the JNS5000, the peak is at the higher frequency of 0.35 . This is consistent with a spectral broadening associated with higher Reynolds number jet flows. At larger frequencies, the solution of Powell's and Lighthill's acoustic analogies diverge somewhat. The solutions of Powell's acoustic analogies appear to contain some spurious energy at these frequencies, which we believe is related to the problem of coordinate dependency of the analogy, as discussed by Avital et al. $(2008)^{21}$.

\section{Data manipulation: acoustic testing}

In this section, we modify the source data in several manners in order to identify crucial dependencies of the predictions on the input source data. The first step in this procedure was to generate a subset of the data on a uniform Cartesian mesh, that might resemble the domain of the PIV data. This was done by a linear interpolation of each of the saved spherical coordinate flow fields onto a mesh with 24 grid points per jet diameter in all dimensions. The resultant grid, which was used for both JNS2500 and JNS5000 can be seen in figure 7 (a), while in figure 7 (b), a 2-D slice of the interpolated axial velocity is shown along with labeled surfaces that bounded acoustic integration domains. We now go through each of the tests and discuss the relevant results in turn.

\section{A. Conversion to regular Cartesian}

In figure 8 (a), we plot the spectra of solutions of Powell's and Lighthill's acoustic analogy using data linearly interpolated into the full Cartesian domain shown in figure 7 (b). What is very evident in these figures is a hump in the high frequency component, after a Strouhal number of 1.0. The most likely cause of this hump is an aliasing of energy related to the linear interpolation into flow modes that generate acoustic waves. To be sure that the effect is not related to the new domain shape, in figure 8 (b), we perform the same computations, but with a sinusoidal window applied to all boundaries of the data. The result is unchanged. 


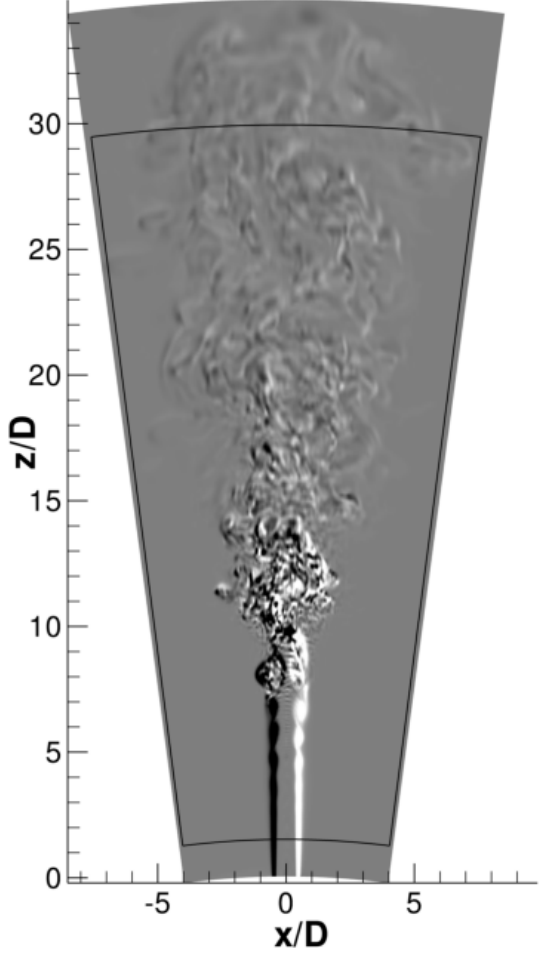

(a)

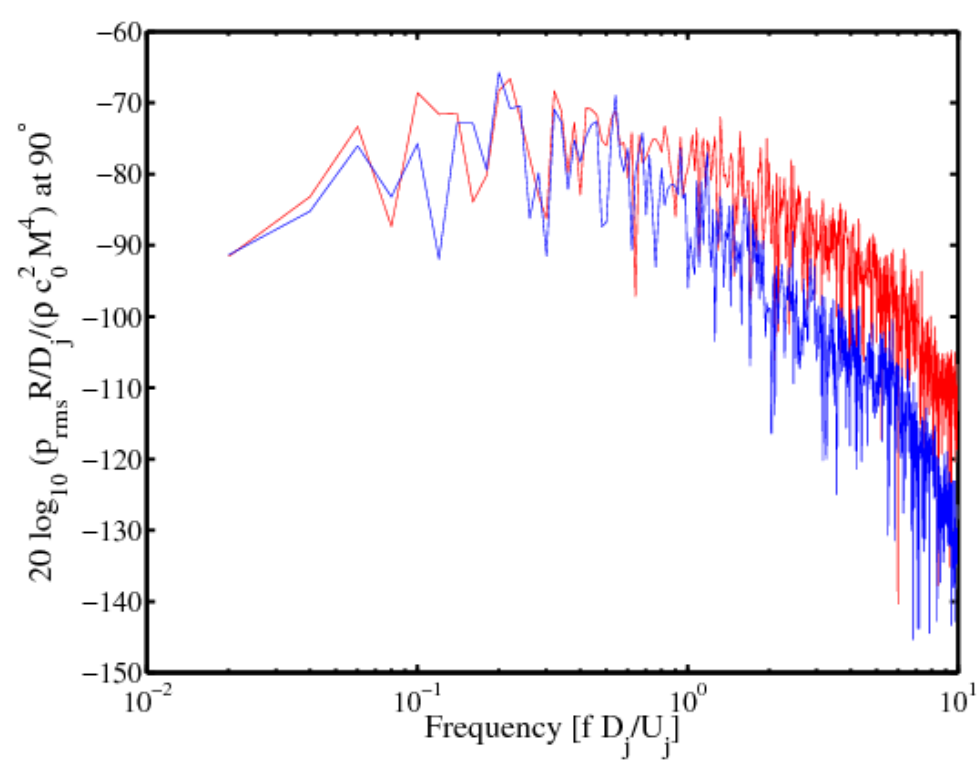

(b)

Figure 5. Acoustic spectra at $90^{\circ}$ for JNS2500 in the full spherical coordinate system. Blue line is Lighthill's acoustic analogy and red line is Powell's acoustic analogy

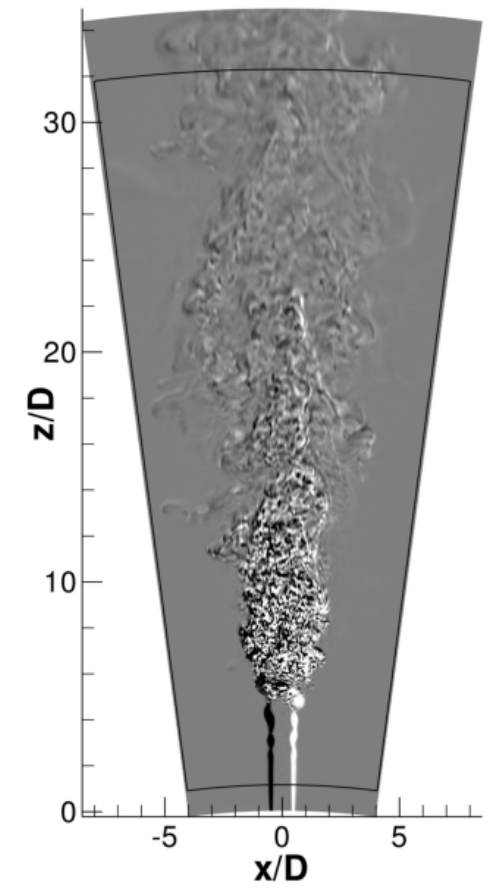

(a)

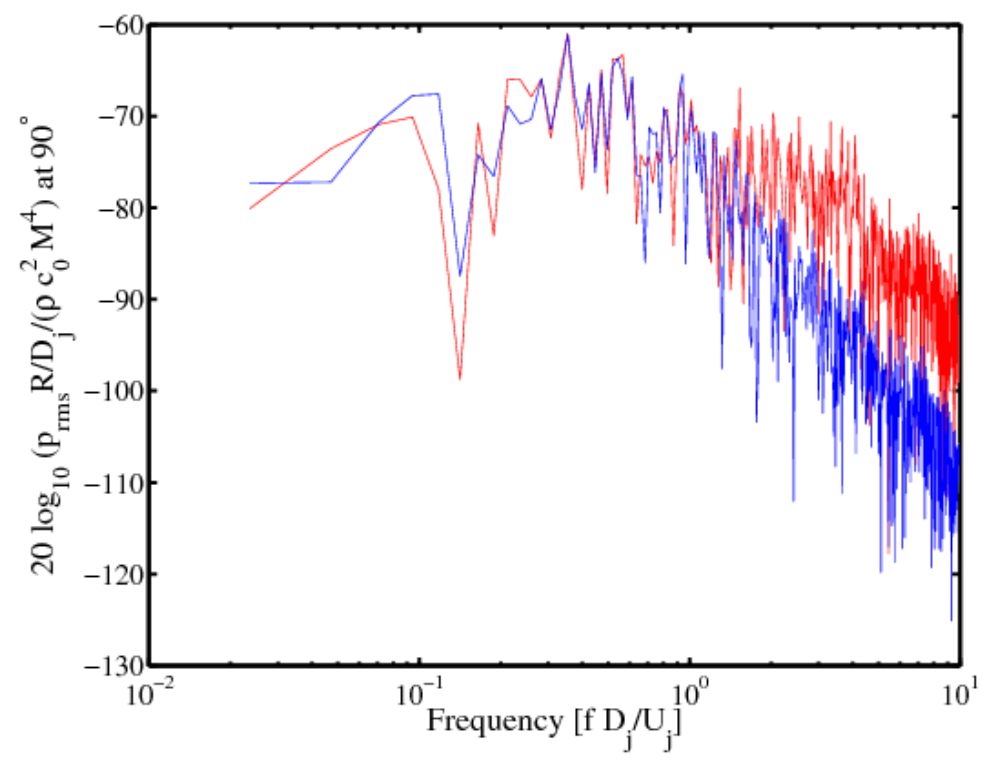

(b)

Figure 6. Acoustic spectra at $90^{\circ}$ for JNS5000 in the full spherical coordinate system. Blue line is Lighthill's acoustic analogy and red line is Powell's acoustic analogy 


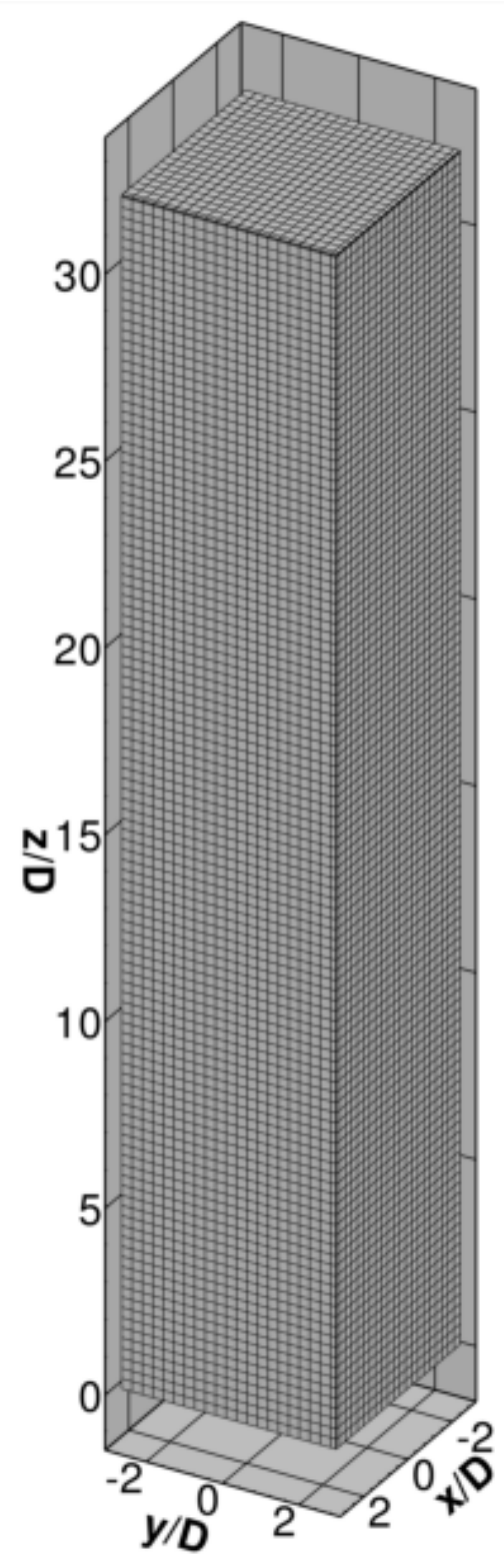

(a)

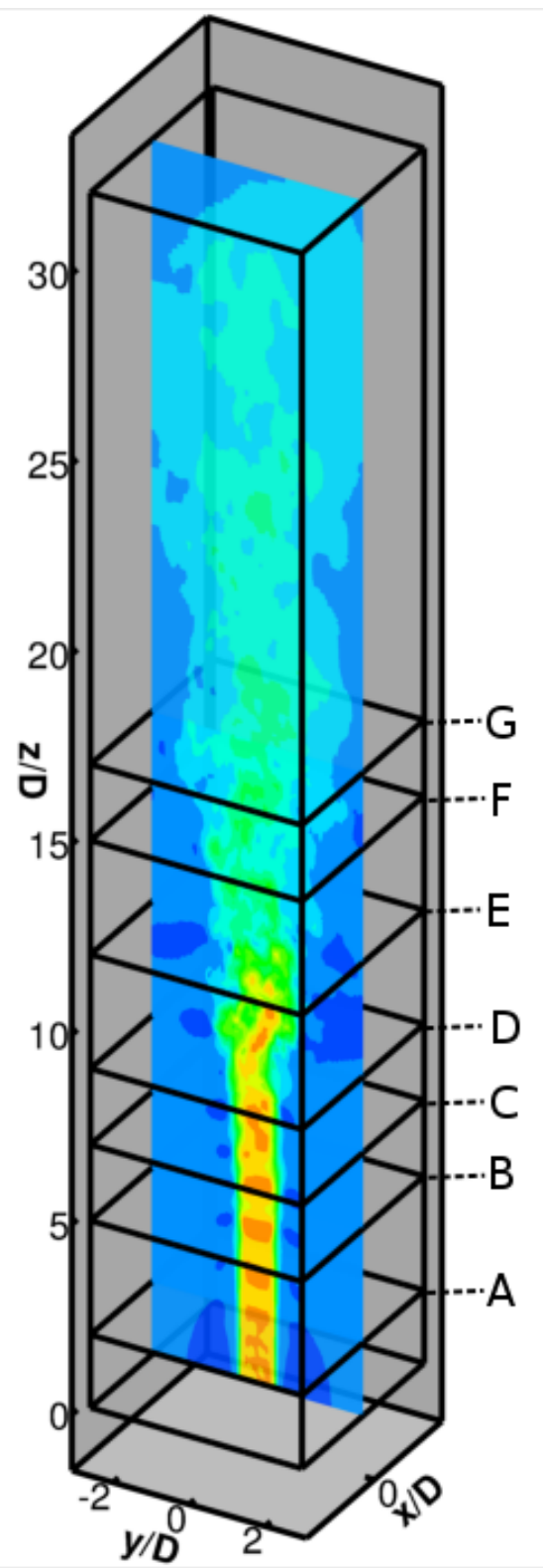

(b)

Figure 7. (a) Cartesian grid used to test dependencies. Every 6th grid line is shown. (b) Integration bounding surfaces. 


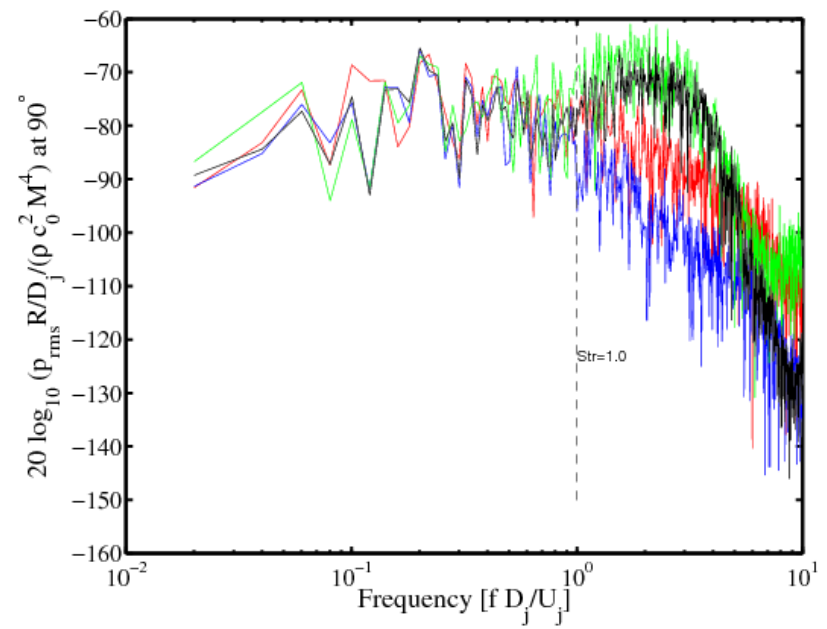

(a)

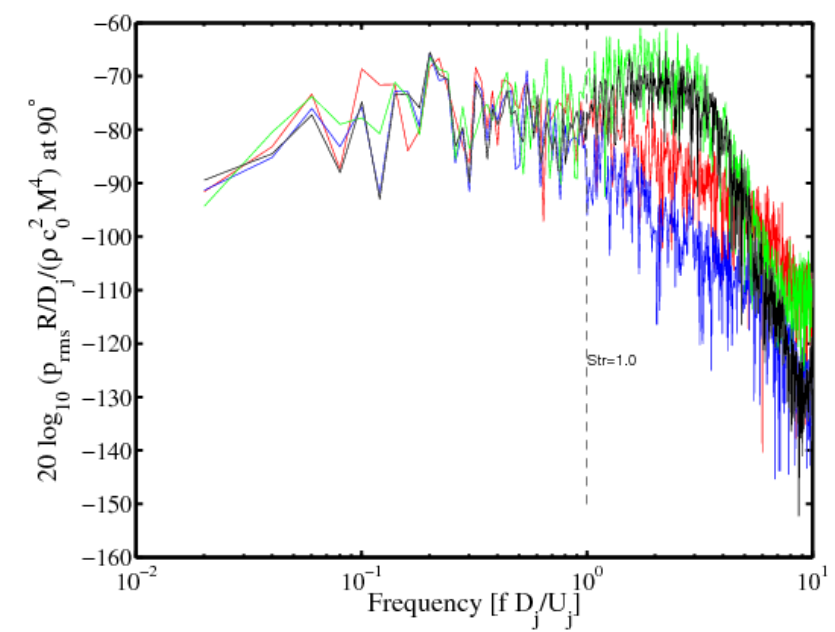

(b)

Figure 8. Acoustic spectra at $90^{\circ}$ for JNS2500 testing conversion to Cartesian coordinates. Blue line is Lighthill's acoustic analogy with original coordinates, red line is Powell's acoustic analogy with original coordinates, black line is Lighthill's in Cartesian coordinates and red line is Powell's in Cartesian coordinates. (a) Without data windowing and (b) with data windowing.

Note that the location of this hump gives a good idea as to what Strouhal number PIV data on such a domain could be trusted to, as measurement uncertainty will also lead to aliasing of energies into acoustic producing modes. Note also that this resolution is quite similar to what is often claimed by LES studies.

\section{B. Time step}

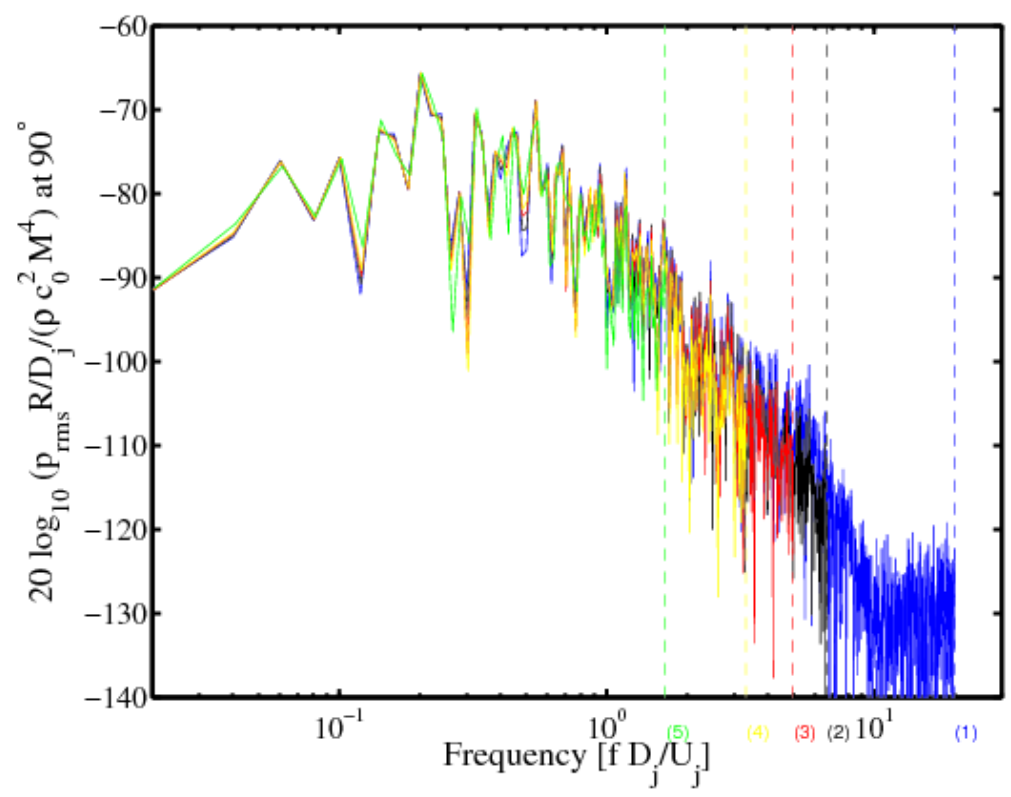

Figure 9. Acoustic predictions by Lighthill's acoustic analogy at $90^{\circ}$ to the jet axis with different time steps. Blue line: $\Delta t \frac{U}{D_{j}}=0.025$, black line: 0.075 , red line: 0.1 , yellow line: 0.15 and green line: 0.3

In figure 9 we perform acoustic predictions by Lighthill's acoustic analogy using the original data set for JNS2500. We test specifically how predictions suffer as the temporal resolution is reduced. Interestingly, lowering the temporal resolution has the expected effect of reducing the frequency cut-off due to the respective Nyquist limits, but has no effect on the frequencies withing the measured range. For the measurements we have already performed, the temporal resolutions achieved are sufficient for predictions to a Strouhal number of 10, and could potentially be reduced by a factor of 10 and without any effect on the frequency range that is unaffected by spatial aliasing. 


\section{Domain reduction}

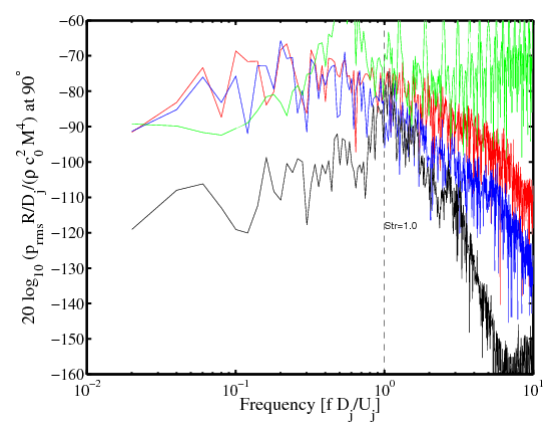

(a) Surface A-B

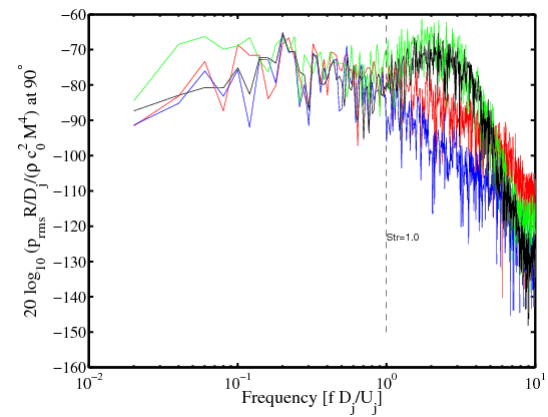

(d) Surface A-E

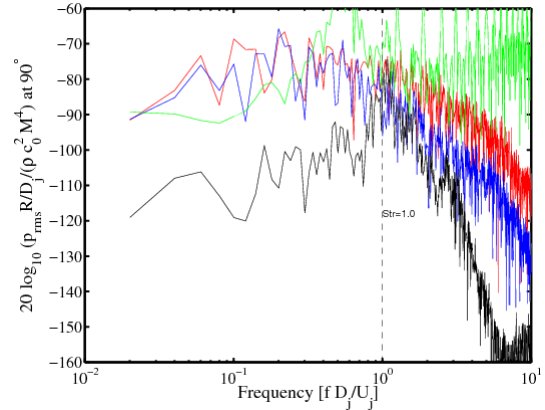

(b) Surface A-C

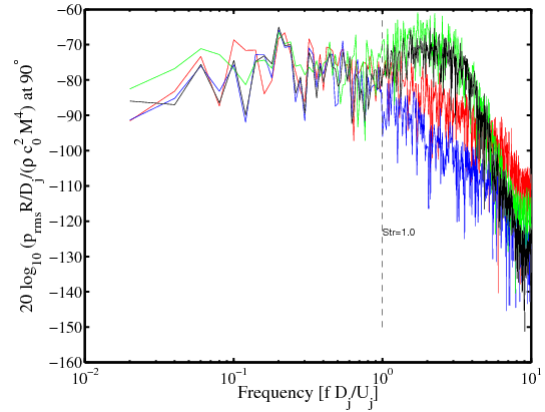

(e) Surface A-F

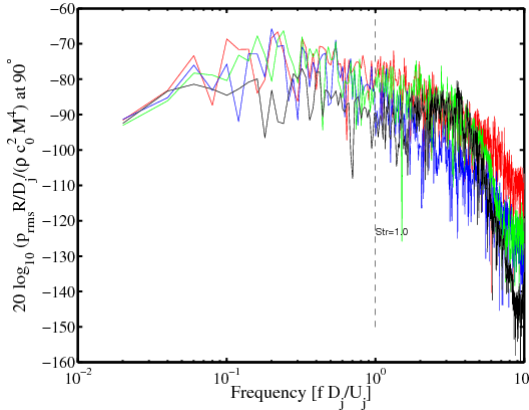

(c) Surface A-D

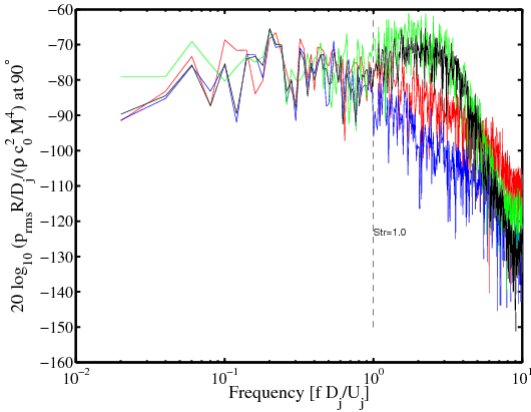

(f) Surface A-G

Figure 10. Acoustic spectra at $90^{\circ}$ for JNS2500. Blue line is Lighthill's acoustic analogy with original coordinates, red line is Powell's acoustic analogy with original coordinates, black line is Lighthill's in Cartesian coordinates and red line is Powell's in Cartesian coordinates.

In figures 10 (a)-(f), we compute solutions of Lighthill's and Powell's acoustic analogies with the Cartesian interpolated data on reduced domains, along with the reference spectra from figure 5. The surface labels refer to the inflow and outflow boundaries that are given in figure 7 (b). The low frequency range is well captured by surface A-E, A-F and A-G, but not A-B, A-C or A-D. The low frequency range is thus captured with a domain length of just $10 D_{j}$. The downstream surface in this case is within just $2 D_{j}$ of the end of the potential core. For TR-TOMO-PIV measurements, this is good news, as the source region can be efficiently localized.

\section{Other angles of emission}

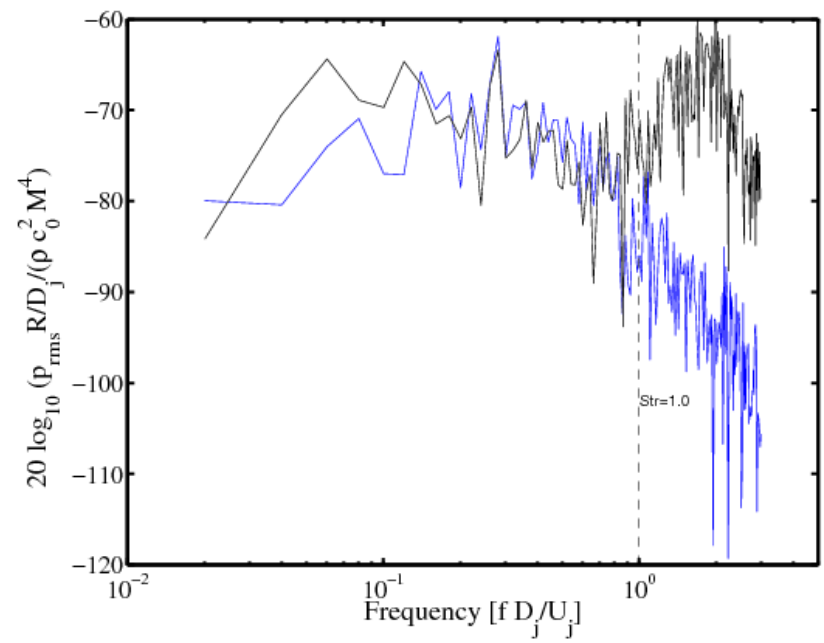

(a) Surface A-E: windowing

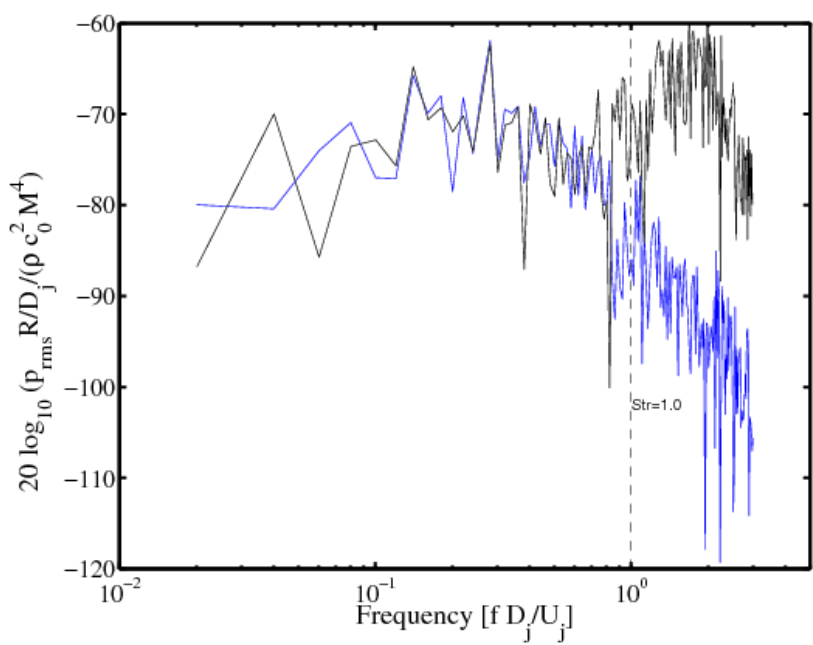

(b) Surface A-F: windowing

Figure 11. Acoustic spectra at $30^{\circ}$ for JNS2500. Blue line is Lighthill's acoustic analogy with original coordinates, black line is Lighthill's in Cartesian coordinates.

In figure 11, we demonstrate briefly that these results are applicable at other angles of emission than $90^{\circ}$. For these 
results, only solutions of Lighthill's acoustic analogies are shown, solutions of Powell's acoustic analogies require origin corrections which are not yet implemented.

\section{E. Reynolds number}

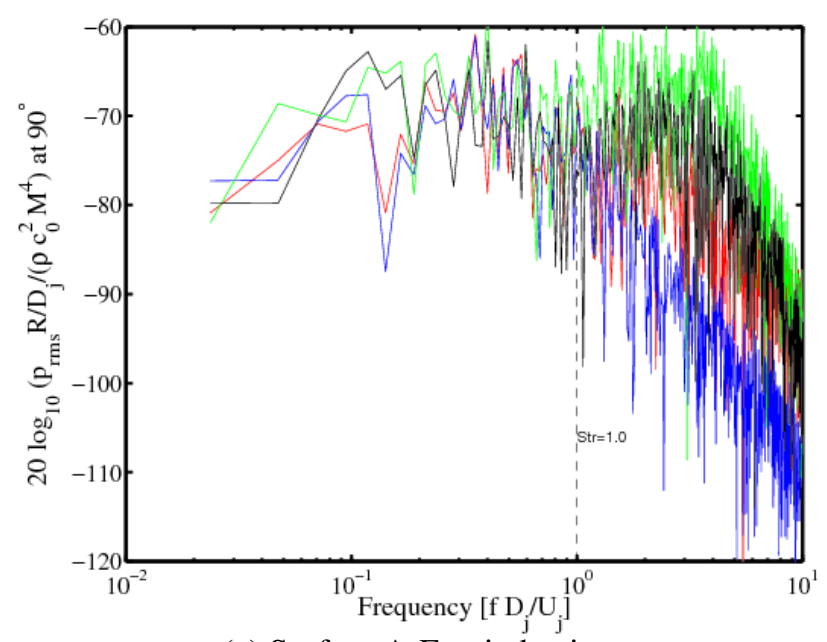

(a) Surface A-E: windowing

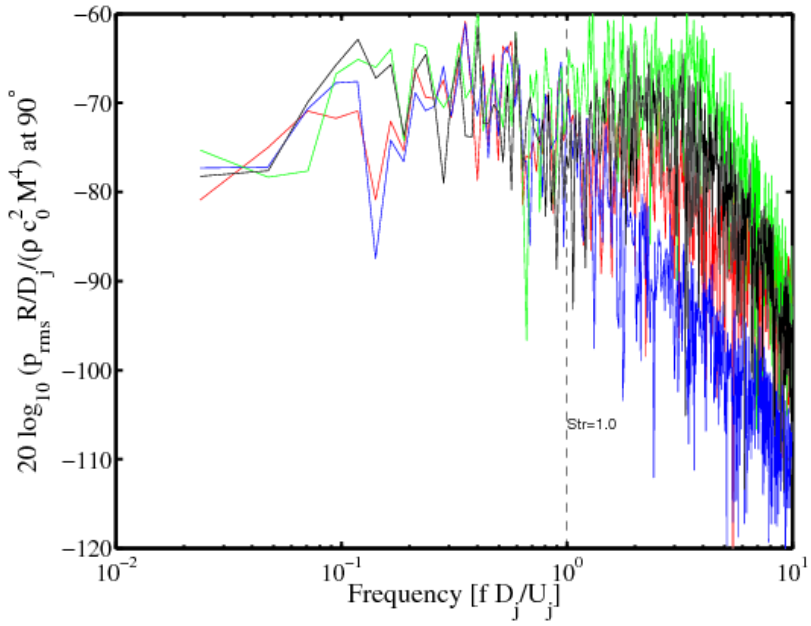

(b) Surface A-E: windowing

Figure 12. Acoustic spectra at $90^{\circ}$ for JNS5000. Blue line uses Lighthill's acoustic analogy with original coordinates, red line uses Powell's acoustic analogy with original coordinates, black line is Lighthill's in Cartesian coordinates and red line is Powell's in Cartesian coordinates.

The last test of this paper is to look at some results for the Reynolds number 5,000 jet JNS5000, and compare with the reference solution from figure 6 . These results appear to indicate that the low frequency range can be similarly captured, although of course at higher Reynolds numbers the missing high frequency component contributes more to the total jet noise.

\section{Conclusions}

3-D Direct Numerical Simulations have been completed for incompressible round jets at Reynolds numbers of 2,500 and 5,000. The conditions of these simulations loosely matched those of experiments undertaken by us of Tomographic PIV in a water tank. The flow statistics and visualizations confirm that the simulated jets are representative of the respective experimental ones. Acoustic predictions on the full jet database are made with Powell's and Lighthill's acoustic analogies.

The effects of limiting temporal information, domain size and spatial resolution were then tested with the aim of determining the minimum requirements for accurate prediction. The results indicate that predictions can be made up to a Strouhal number of 1.0 with resolutions similar to that achievable by our PIV system. The results identify that the necessary temporal resolution is easily achieved, while higher spatial resolution should be obtained at the expense of domain size in order to make successful predictions.

Finally, the effects of experimental uncertainty have not yet been tested, but if satisfactory methods can be demonstrated to resolve any issues with experimental uncertainty, then acoustic prediction by TR-TOMO-PIV is achievable with our current equipment for these jet flows.

\section{Acknowledgments}

This work was conducted as part of the FLOVIST project (2008-2013), funded by the European Research Council, European Commission "Ideas" FP7 Programme. The authors also acknowledge the use of code from Bendiks Jan Boersma for the jet simulations. 


\section{References}

${ }^{1}$ Elsinga, G.E. amd Wieneke, B., Scarano, F., and Schröder, A., "Tomographic 3D-PIV and Applications," Particle Image Velocimetry, Topics in Applied Physics, Vol. 112, 2008, pp. 103-125.

${ }^{2}$ Koschatzky, V., Delfos, R., Boersma, B. J., and Westerweel, J., "Boundary layer influence on cavity noise generation," Advances in Turbulence

XII, Proceedings of the 12th EUROMECH European Turbulence Conference, September 7-10, 2009, Marburg, Germany, Vol. $132,2009$.

${ }^{3}$ Haigermoser, C., "Application of an acoustic analogy to PIV data from rectangular cavity flows," Experiments in Fluids, 2009.

${ }^{4}$ Curle, N., "The influence of solid boundaries upon aerodynamic sound," Proc. Roy. Soc. Lon., Vol. A 231, 1955, pp. 505-514.

${ }^{5}$ Lorenzoni, V., Tuinstra, M., Moore, P., and Scarano, F., "Aeroacoustic analysis of a rod-airfoil flow by means of time-resolved PIV," 15th AIAA/CEAS Aeroacoustics Conference, Miami, Florida, 2009.

${ }^{6}$ Moore, P., Lorenzoni, V., and Scarano, F., "Comparison of two techniques for aeroacoustic determination from a rod-airfoil PIV experiment," 8th International Symposium on Particle Image Velocimetry, Melbourne, Australia, 2009.

${ }^{7}$ Wernet, M., "Temporally resolved PIV for space-time correlations in both hot and cold jet flows," Measurement Science and Technology, Vol. 18, 2007, pp. 1387-1403.

${ }^{8}$ Schram, C. and Hirshberg, A., "Application of vortex sound theory to vortex-pairing noise: sensitivity to errors in flow data," Journal of Sound and Vibration, Vol. 266, no. 5, 2003, pp. 1079-1098.

${ }^{9}$ Schram, C., Taubitz, S., Anthoine, J., and Hirshberg, A., "Theoretical / empirical prediction and measurement of the sound produced by vortex pairing in a low Mach number jet," Journal of Sound and Vibration, Vol. 281(1-2), 2005, pp. 171-187.

${ }^{10}$ Stromberg, J., Mclaughlin, D., and Troutt, T., "Flow Field and Acoustic Properties of a Mach Number 0.9 Jet at a Low Reynolds Number," Journal of Sound and Vibration, Vol. 72, 1980, pp. 159-176.

${ }^{11}$ Jordan, P. and Gervais, Y., "Subsonic jet aeroacoustics: associating experiment, modelling and simulation," Experiments in Fluids, Vol. 44, 2008, pp. 1-21.

${ }^{12}$ Violato, D., Bryon, K., Moore, P., and Scarano, F., "Application of Powell's analogy for the prediction of vortex-pairing sound in a low-Mach number jet based on time-resolved planar and tomographic PIV," 16th AIAA/CEAS Aeroacoustics Conference, Stockholm, Sweden., 2010.

${ }^{13}$ Gröschel, E., Meinke, M., and Schröder, W., "Noise prediction for a turbulent jet using an LES/CAA method," 11th AIAA/CEAS Aeroacoustics Conference, Vol. 3039, 2005.

${ }^{14}$ Boersma, B., Brethouwer, G., and Nieuwstadt, F., "A numerical investigation on the effect of the inflow conditions on the self-similar region of a round jet," Physic. Fluids, Vol. 10, 1998, pp. 899-909.

${ }^{15}$ Michalke, A., "Survey on jet instability theory," Prog. Aerospace Sci., Vol. 21, 1984, pp. 159-199.

${ }^{16}$ Freund, J., "Noise sources in a low-Reynolds-number turbulent jet at Mach 0.9," J. Fluid Mech., Vol. 438, 2001, pp. 277-305.

${ }^{17}$ Lighthill, M., "On sound generated aerodynamically, Part 1: General theory," Proceedings of the Royal Society of London, Vol. A 211, 1952, pp. 564-587.

${ }^{18}$ Powell, A., "Theory of vortex sound," Journal of the Acoustical Society of America, Vol. 36 n. 1, 1964, pp. 177-195.

${ }^{19}$ Wang, M., Lele, S., and Moin, P., "Computation of Quadrupole Noise Using Acoustic Analogy," AIAA Journal, Vol. 34, No. 11, 1996.

${ }^{20}$ Avital, E., Sandham, N., and Luo, K., "Calculation of basic sound radiation of axisymmetric jets by Direct Numerical Simulations," AIAA Journal, Vol. 37, No. 2, 1999.

${ }^{21}$ Avital, E., Alonso, M., and Suponitsky, V., "Computational aeroacoustics: The low speed jet," The Aeronautical Journal, Vol. 112, No. 1113, 2008.

${ }^{22}$ Obrist, D. and Kleiser, L., "The influence of spatial domain truncation on the prediction of acoustic far-fields," 13th AIAA/CEAS Aeroacoustics Conference, Vol. 3725, 2007. 